\title{
BMJ Open Sugar-sweetened beverage consumption, correlates and interventions among Australian Aboriginal and Torres Strait Islander communities: a scoping review protocol
}

Jodie C Avery, ${ }^{1,2}$ Jacqueline A Bowden, ${ }^{1,3}$ Joanne Dono, ${ }^{1}$ Odette R Gibson, ${ }^{4}$ Aimee Brownbill, ${ }^{1}$ Wendy Keech, ${ }^{4}$ David Roder, ${ }^{5}$ Caroline L Miller ${ }^{1,6}$

To cite: Avery JC, Bowden JA, Dono J, et al. Sugar-sweetened beverage consumption, correlates and interventions among Australian Aboriginal and Torres Strait Islander communities: a scoping review protocol. BMJ Open 2017;7:e016431. doi:10.1136/ bmjopen-2017-016431

\section{- Prepublication history for} this paper is available online. To view these files please visit the journal online (http://dx.doi. org/10.1136/bmjopen-2017016431).

Received 15 February 2017

Revised 1 May 2017

Accepted 13 June 2017

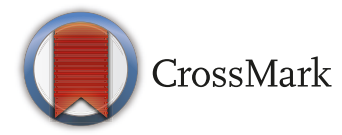

For numbered affiliations see end of article.

Correspondence to Dr Jodie C Avery; jodie.avery@adelaide.edu.au

\section{ABSTRACT}

Introduction Aboriginal and Torres Strait Islander communities of Australia experience poorer health outcomes in the areas of overweight and obesity, diabetes and cardiovascular disease. Contributing to this burden of disease in the Australian community generally and in Aboriginal and Torres Strait Islander communities, is the consumption of sugar-sweetened beverages (SSBs). We have described a protocol for a review to systematically scope articles that document use of SSBs and interventions to reduce their consumption with Aboriginal and Torres Strait Islander people. These results will inform future work that investigates interventions aimed at reducing harm associated with SSB consumption.

Methods and analysis This scoping review draws on a methodology that uses a six-step approach to search databases including PubMed, SCOPUS, CINAHL, Informit (including Informit: Indigenous Peoples), Joanna Briggs Institute EBP Database and Mura, between January 1980 and February 2017. Two reviewers will be engaged to search for and screen studies independently, using formulated selection criteria, for inclusion in our review. We will include primary research studies, systematic reviews including meta-analysis or meta-synthesis, reports and unpublished grey literature. Results will be entered into a table identifying study details and characteristics, summarised using a Preferred Reporting Items for Systematic Reviews and Meta-Analysis chart and then critically analysed.

Ethics and dissemination This review will not require ethics committee review. Results will be disseminated at appropriate scientific meetings, as well as through the Aboriginal and Torres Strait Islander community.

\section{INTRODUCTION}

Sugar-sweetened beverages (SSBs) are increasingly becoming a global concern due to the adverse health effects associated with consumption. Recent systematic reviews and a meta-analysis have supported a causal association between SSB intake and weight gain/obesity. ${ }^{12}$ SSB consumption has also

\section{Strengths and limitations of this study}

- The review is important to consolidate the evidence in this area, identifying research gaps that may contribute to addressing Aboriginal and Torres Strait Islander health.

- We have been guided by the principles of the South Australian Aboriginal Research Accord in formulating this protocol.

- Scoping reviews are suitable when the purpose is to map key concepts within a research area as well as to clarify working definitions; however, they are not as precise as a systematic review to answer a research question.

- As the mapping of the research evolves through the process of the review, we cannot be completely specific about our definitions until the actual scoping review is undertaken

been identified as an independent risk factor for non-communicable diseases including type II diabetes, cardiovascular disease and some cancers. ${ }^{3} 4$ Additionally, consumption is linked to dental caries. ${ }^{56}$ As there is a clear link between SSB consumption and poor health outcomes, reducing consumption of these products on a population level, through supportive environments that shape food choice, is one way to address obesity and obesity-related non-communicable diseases. ${ }^{78}$

Consumption of SSBs is higher among Aboriginal and Torres Strait Islander people than in the wider population. Half of Aboriginal and Torres Strait Islander people (50\%) consumed SSBs on the day prior to the Australian Health Survey in 2011-2012 compared with $34 \%$ of non-indigenous people. ${ }^{9} \mathrm{Com}-$ pared with non-indigenous people, Aboriginal and Torres Strait Islander people also experience higher rates of overweight/obesity with 


\begin{tabular}{|c|c|}
\hline Topic & Search terms \\
\hline SSBs & 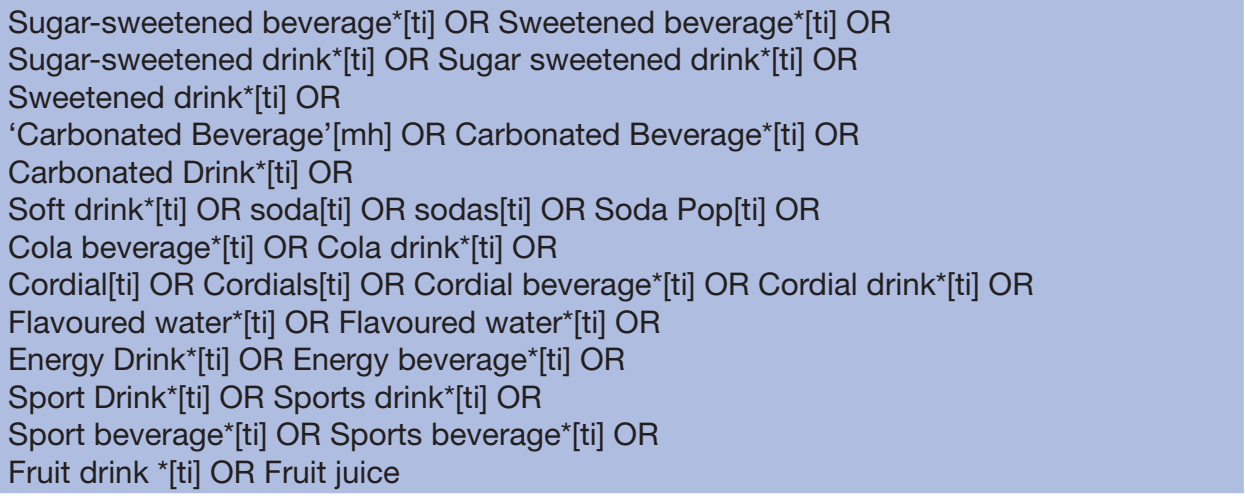 \\
\hline Interventions ${ }^{\star}$ & $\begin{array}{l}\text { Intervention [tiab] OR Strategy [tiab] OR Taxation [tiab] OR Education [tiab] OR Social } \\
\text { Marketing [tiab] }\end{array}$ \\
\hline $\begin{array}{l}\text { Aboriginal and Torres Strait Islander } \\
\text { and other indigenous populations }\end{array}$ & $\begin{array}{l}\text { Aboriginal*[tiab] OR Indigenous[tiab] OR } \\
\text { 'Torres Strait Islander*'[tiab] OR } \\
\text { Aborigines, Australian [tiab] OR Aborigine, Australian [tiab] OR Australian Aborigine [tiab] } \\
\text { OR Australian Aborigines [tiab] OR Natives [tiab] OR Native-Born [tiab] OR Native Born } \\
\text { [tiab] OR Indigenous Population [tiab] OR Indigenous Populations [tiab] OR Population, } \\
\text { Indigenous [tiab] OR Populations, Indigenous [tiab] }\end{array}$ \\
\hline NOT & $\begin{array}{l}\text { Non-nutritive sweeteners[mh] OR Non-nutritive sweetener*[tiab] OR } \\
\text { Artificial sweetener*[tiab] OR Artificially sweetened[tiab] OR } \\
\text { Alcoholic beverages[mh] OR Alcohol[tiab] } \\
\text { OR } \\
\text { Alcoholic beverage*[tiab] OR Alcoholic drink*[tiab] }\end{array}$ \\
\hline
\end{tabular}

*Will be refined iteratively once initial searches are complete.

$66 \%$ of adults and $29.7 \%$ of children currently overweight or obese. ${ }^{10}$ Furthermore, non-communicable diseases associated with SSB consumption such as diabetes and cardiovascular disease are also disproportionately experienced by Aboriginal and Torres Strait Islander people compared with non-indigenous Australians. ${ }^{10}$

Understanding the relationship between food choices and chronic diseases such as diabetes, cardiovascular disease and obesity was identified as a priority for future research in the 'Next Steps' research project. ${ }^{11}$ This research project was a community-driven approach to identifying research areas that align with the needs and interests of Aboriginal and Torres Strait Islander people in South Australia. The Next Steps document, along with the South Australian Aboriginal Health Research Accord, ${ }^{12}$ was instrumental in the development of an advisory group to explore research options further. Initial discussions from the advisory group established that research on SSB consumption in Aboriginal and Torres Strait Islander communities was a priority and the development of a research plan could build on existing relationships that researchers in the advisory group had developed with the community.

SSB consumption, as a unique contributor to poor health outcomes, is an emerging national health issue in Australia. To reduce SSB consumption, a comprehensive, multi-level strategy is needed because of the widespread availability and demand for these products. Exploring the potential outcomes of interventions designed to reduce SSB consumption is important to ensure that they do not reinforce or create health inequalities. Aboriginal and Torres Strait Islander people compared with non-indigenous Australians experience higher socio-economic disadvantage, have higher exposure to behavioural and environmental health risk factors, have higher proportions of households living in conditions that do not support good health, fewer complete year 12 education and have lower rates of employment. ${ }^{13}$ Additionally, Aboriginal and Torres Strait Islander people view health in a holistic context, the needs of which are not met by the dominant biomedical model of healthcare in Australia. Aboriginal and Torres Strait Islander people have also experienced hardship, dispossession of culture and land and racism, which has contributed to health inequalities and exclusion from the health system. ${ }^{11}$ Therefore, we expect that interventions that are community driven and take into account the specific needs of a community may be more effective than interventions targeted to the general Australian population. ${ }^{14}$

In order to address SSB consumption as a chronic disease research priority, it was decided to first undertake a scoping review to identify the scope and nature of the literature that documents the consumption of SSBs and interventions to reduce their consumption with Australian Aboriginal and Torres Strait Islander people. 


\section{METHODS}

An initial search of major databases found no reviews concerning our research question. The refined scoping review framework was selected. ${ }^{15} 16$ This framework is appropriate where the objective is to understand and map the literature in a systematic and transparent manner and includes six steps: identification of relevant studies, selection of studies, charting of the data, collating, summarising and reporting of the articles and consultation or knowledge translation. ${ }^{16}$

\section{Stage 1: identification of the research question}

We used the 'PICo' framework to define our Population $(\mathrm{P})$, phenomenon of Interest (I) and Context (Co) for our research question. ${ }^{15}$ Our population of interest is the Australian Aboriginal and Torres Strait Islander communities within the context of the general Australian population. Although SSB consumption is highest in those aged 30 and under, consumption is high across the life course ${ }^{10}$; therefore all ages will be included within the review. The phenomenon of interest is strategies aimed at reducing the consumption of SSBs. Our Context includes the various intervention approaches available and the next most appropriate course of action to reduce the risk of harm from consuming SSBs in Australian Aboriginal and Torres Strait Islander communities.

\section{Scoping review question}

The aim of this scoping review is to rapidly identify the scope and nature of the current literature that documents the consumption of SSBs and interventions to reduce their consumption with Australian Aboriginal and Torres Strait Islander people. It will address the following questions:

- Which demographics, within the Aboriginal and Torres Strait Islander communities, are consuming the most SSBs and does this differ across communities?

- What are the social and environmental conditions that influence SSB consumption in Aboriginal and Torres Strait Islander communities?

- What interventions that aim to reduce SSB consumption in Aboriginal and Torres Strait Islander communities have been implemented and evaluated?

- What interventions that aim to reduce SSB consumption in the broader Australian population have been implemented and evaluated and to what extent have the implications for Aboriginal and Torres Strait Islander communities been explored?

These research questions were developed with an advisory group including the chief investigators of the project, researchers from the Population Health Research Group and the Wardliparingga Aboriginal Research Unit at the South Australian Health and Medical Research Institute.

\section{Stage 2: identifying relevant studies}

The primary databases included for searching for this review include PubMed, SCOPUS, CINAHL, Informit, Informit: Indigenous Peoples, Joanna Briggs Institute EBP Database and Mura. Databases will be searched using the keywords and index terms specified in table 1, and sources found between January 1980 and February 2017 will be included to reflect the growing interest in this area. Reference lists of relevant articles will be searched for additional records. Grey literature searches will be completed using the Google search engine, ProQuest (for dissertations), the Australian Policy Online research database (http://www.apo.org.au) and the Australian Indigenous Health Info Net database (http://www. healthinfonet.ecu.edu.au). Search terms are likely to evolve during searching in an iterative process. The initial terms will be: sugar-sweetened beverages, soft drinks, cola, cordial, flavoured water, energy drink, sports drinks, Aboriginal, indigenous, Australia, interventions and strategies. For each combination of search terms, results will be scanned for items of interest until saturation occurs (no new items within two search results pages). The web browser's bookmark and folders functions will be used to save pages of interest, organise results by search terms and detect duplicates. Relevant records will be exported to EndNote. A record will be kept of search terms used, and number of results scanned and retrieved. Existing networks, relevant organisations and conferences will also be considered. The data will be managed using EndNote $\mathrm{X} 7.5 .^{17}$

A preliminary search in PubMed, SCOPUS, the University of Adelaide Library and Google Scholar using search terms described in table 1 confirmed that this review has not been done.

\section{Stage 3: study selection (screening)}

In selecting studies to include as part of this scoping review, a primary reviewer will remove duplicates and then scan titles and abstracts to further remove any irrelevant articles. With the help of a second reviewer, full articles will be read to determine eligibility by applying the inclusion/exclusion criteria. Relevant information will be extracted from included articles.

\section{Inclusion criteria}

\section{Participants and context}

Aboriginal and Torres Strait Islander people within the context of the Australian population. All age groups will be eligible for inclusion. This scoping review has adopted the United Nations definition of indigenous people, "descendants of those who inhabited a country or a geographical region at the time when people of different cultures or ethnic origins arrived'. ${ }^{18}$

\section{Concepts and definitions}

- SSBs: the National Health and Medical Research Council recommends in the Australian Dietary Guidelines limiting the intake of sugar-sweetened soft drinks and cordials, fruit drinks, vitamin waters, energy and sports drinks. ${ }^{19}$

- Interventions: any attempt to reduce risk from harms associated with SSB consumption. Interventions that have been trialled in Aboriginal and Torres Strait Islander communities will be examined. In addition, potential interventions identified by the Centres for 
Disease Control will be eligible such as ensuring ready access to potable drinking water, limiting access to SSBs, promoting access to and consumption of more healthful alternatives to SSBs, limiting marketing of SSBs and minimising marketing's impact on children, decreasing the relative cost of more healthful beverage alternatives through differential pricing of SSBs, including screening and counselling about SSB consumption as part of routine medical care and expanding the knowledge and skills of medical care providers to conduct nutrition screening and counselling regarding SSB consumption, ${ }^{20}$ as well as 'nudging,' which includes reducing portion size, changing the shape of the drink containers and changing the shelf location of SSBs. ${ }^{21}$

\section{Sources and types of studies}

Existing primary research studies, of both quantitative and qualitative design, systematic reviews including meta-analysis or meta-synthesis, reports and unpublished grey literature.

\section{Exclusion criteria}

Studies will be sorted by country, and only those conducted in Australia will be included. Studies that have an emphasis on sugar in foods rather than specifically SSBs and publications not available in English will be excluded due to cost and time in translation. Interventions will be excluded if they do not include at least one component that aims to reduce SSB consumption or do not have a specific measure of a construct related to SSB consumption (eg, health knowledge, or attitudes towards, SSBs).

\section{Stage 4: charting the evidence (data abstraction)}

A copy of each article will be stored in an EndNote $^{17}$ database and the following study details and characteristics will be extracted and entered into a table:

- Year of publication

- Location: country/community/urban/rural/ remote

- Type of intervention

- Number of participants

- Age range

- Sex

- Aim

- Study type (eg, lab experiment, field experiment, prepost survey, longitudinal survey, cross-sectional survey)

- Source (eg, case report, letters, primary research)

- Methodology/methods

- Intervention type, comparator and details of these (eg, duration of the intervention) (if applicable)

- Scope (eg, how it addresses our research question)

- Results (eg, consumption prevalence or a measure of intervention impact)

- Limitations

- Conclusions
Stage 5: collating, summarising and reporting the data

The search process will be summarised using a Preferred Reporting Items for Systematic Reviews and Meta-Analysis chart. ${ }^{22}$ The type of studies will be described, and as this is a scoping review, results from the selected studies will be presented in a narrative form highlighting themes found throughout the literature.

\section{Stage 6: consultation (knowledge translation)}

The approach to consultation and knowledge translation is informed by the South Australian Aboriginal Health Research Accord's nine principles by which Aboriginal and Torres Strait Islander research should be conducted. ${ }^{12}$ In accordance with these principles, the research questions are based on priorities identified by indigenous communities, and the research advisory group includes members of the Wardliparingga Aboriginal Research Unit of the South Australian Health and Medical Research Institute. A strengths-based approach will be taken throughout the review. The draft scoping review report will be reviewed by the entire research advisory group. The dissemination plan for the results of the review depends, in part, on the research findings. Following the review of the draft, the Wardliparingga Aboriginal Research Unit will provide advice and assist in disseminating the results to other key indigenous organisations and community elders, prior to publication. Open-access publication will be sought.

\section{ETHICS AND DISSEMINATION}

This review will not require ethics committee review. We will disseminate the results of this review through publication in a peer-reviewed journal, and at appropriate scientific meetings, as well as through the Aboriginal and Torres Strait Islander community. Data from this review will be summarised in a table published alongside the full review.

\section{CONCLUSION}

The key outputs from this review will be an understanding of past and current efforts in reducing SSB consumption among Australian indigenous populations. These results will inform future work investigating the availability, consumption and interventions aimed at reducing harm associated with consumption SSBs among Aboriginal and Torres Strait Islander Communities within the context of the broader Australian population.

Author affiliations
${ }^{1}$ Population Health Research Group, South Australian Health and Medical
Researchlnstitute, Adelaide, Adelaide, South Australia, Australia
${ }^{2}$ School of Medicine, University of Adelaide, Adelaide, South Australia, Australia
${ }^{3}$ School of Psychology, University of Adelaide, Adelaide, South Australia, Australia
${ }^{4}$ Wardliparingga Aboriginal Research Unit, South Australian Health and
MedicalResearch Institute, Adelaide, South Australia, Australia
${ }^{5}$ Centre for Population Health Research, University of South Australia, Adelaide,
South Australia, Australia
${ }^{6}$ School of Public Health, University of Adelaide, Adelaide, South Australia, Australia


Contributors JCA is the guarantor. JAB, CLM and DR conceived the idea for the project. JCA drafted the manuscript. $A B$ assisted with the preliminary literature search. JCA, JAB, JD and CLM developed the research question. JD and CLM provided expertise on sugar sweetened beverages. ORG and WK provided expertise in the area of Aboriginal and Torres Strait Islander research, the relevance of the research direction and method to answer the question. All authors contributed to the development of the selection criteria. All authors read, provided feedback and approved the manuscript.

Funding This work was supported by the University of South Australia and the NHMRC Program Grant no. 631947.

Competing interests None declared.

Provenance and peer review Not commissioned; externally peer reviewed.

Open Access This is an Open Access article distributed in accordance with the Creative Commons Attribution Non Commercial (CC BY-NC 4.0) license, which permits others to distribute, remix, adapt, build upon this work non-commercially, and license their derivative works on different terms, provided the original work is properly cited and the use is non-commercial. See: http://creativecommons.org/ licenses/by-nc/4.0/

(C) Article author(s) (or their employer(s) unless otherwise stated in the text of the article) 2017. All rights reserved. No commercial use is permitted unless otherwise expressly granted.

\section{REFERENCES}

1. Te Morenga L, Mallard S, Mann J. Dietary sugars and body weight: systematic review and meta-analyses of randomised controlled trials and cohort studies. BMJ 2012;346:e7492.

2. Malik VS, Pan A, Willett WC, et al. Sugar-sweetened beverages and weight gain in children and adults: a systematic review and metaanalysis. Am J Clin Nutr 2013;98:1084-102.

3. Vartanian LR, Schwartz MB, Brownell KD. Effects of soft drink consumption on nutrition and health: a systematic review and metaanalysis. Am J Public Health 2007;97:667-75.

4. Malik VS, Popkin BM, Bray GA, et al. Sugar-sweetened beverages and risk of metabolic syndrome and type 2 diabetes: a metaanalysis. Diabetes Care 2010;33:2477-83.

5. Bernabé E, Vehkalahti MM, Sheiham A, et al. Sugar-sweetened beverages and dental caries in adults: a 4-year prospective study. $J$ Dent 2014;42:952-8.

6. Sohn W, Burt BA, Sowers MR. Carbonated soft drinks and dental caries in the primary dentition. J Dent Res 2006;85:262-6.
7. Hu FB. Resolved: there is sufficient scientific evidence that decreasing sugar-sweetened beverage consumption will reduce the prevalence of obesity and obesity-related diseases. Obes Rev 2013;14:606-19.

8. Singh GM, Micha R, Khatibzadeh S, et al. Estimated global, regional, and national disease burdens related to sugar-sweetened beverage consumption in 2010. Circulation 2015;132:639-66.

9. Australian Bureau of Statistics. Australian Aboriginal and Torres Strait Islander Health Survey: First Results, Australia, 2012-13. Canberra: Australia Bureau of Statistics, 2013.

10. Australian Bureau of Statistics. Australian Health survey: nutrition first results-Foods and Nutrients, 2011-12. Table 18: Consumption of Sweetened Beverages. Canberra: Australian Bureau of Statistics, 2015.

11. King R, Brown A. Next steps for Aboriginal Health Research: exploring how research can improve the health and wellbeing of aboriginal people in South Australia. Adelaide: Aboriginal Health Council of South Australia, 2015.

12. Morey K. South Australian Aboriginal Health Research Accord: Companion Document. Adelaide: Wardliparingga Aboriginal Research Unit, South Australian Health and Medical Research Institute, 2014

13. Australian Health Ministers' Advisory Council. Aboriginal and Torres Strait Islander Health Performance Framework 2014 Report. Canberra: AHMAC, 2015.

14. Australia C. National Aboriginal and Torres Strait Islander Health Plan 2013-2023. Canberra 2013.

15. Arksey H, O'Malley L. Scoping studies: towards a methodological framework. Int J Soc Res Methodol 2005;8:19-32.

16. Levac D, Colquhoun H, O'Brien KK. Scoping studies: advancing the methodology. Implement Sci 2010;5:69.

17. Reuters T. EndNote X7.5 program. 2016. 1988.

18. Secretariat of the permanent forum on indigenous issues. The concept of indigenous peoples. Workshop on data collection and disaggregation for indigenous peoples. New York: United Nations, 2004.

19. NHMRC. Australian dietary guidelines. Canberra: National Health and Medical Research Council, 2013

20. Centres for Disease Control. The CDC guide to strategies for reducing the consumption of sugar-sweetened beverages. 2010.

21. Petrescu DC, Hollands GJ, Couturier DL, et al. Public acceptability in the UK and USA of nudging to reduce obesity: the example of reducing sugar-sweetened beverages consumption. PLoS One 2016;11:e0155995.

22. Moher D, Liberati A, Tetzlaff J, et al. Preferred reporting items for systematic reviews and meta-analyses: the PRISMA statement. PLoS Med 2009;6:e1000097. 